\title{
Study on the Teaching of College English Reading Comprehension Based on Schema Theory
}

\author{
H.M. Zhao \\ Teaching and Research Institute of Foreign Languages \\ Bohai University \\ Jinzhou, China
}

\begin{abstract}
Due to the coming of knowledge economy, the lifelong learning becomes necessary for the college students. Currently, the college English education still stays in the traditional teaching mode. This mode does not pay much attention to the cultivation of English reading comprehension ability. The college graduates commonly have low reading comprehension ability and cannot read the professional literatures, and therefore, they cannot achieve new knowledge by themselves. In this paper, the author discusses the cognitive rules of schema theory and applies them to the English teaching of reading comprehension. The author also proposes some strategies on reading comprehension teaching.
\end{abstract}

Keywords-schema theory; reading comprehension; English ability; reform of teaching

\section{INTRODUCTION}

With the coming of knowledge economy, the social demands for personnel are much stricter than ever. The knowledge achieved by students in college is far from meeting the needs of workplace. Therefore, lifelong learning becomes the current problems encountered by college graduates. As an important way to obtain information, reading ability is one of the important abilities cultivated in college English teaching. Currently, college English education still stays in the traditional teaching mode and does not pay attention to the cultivation of reading ability of students. The class of reading even becomes the continuation of grammar and vocabulary teaching. Therefore, the reading ability of students is very low and they are usually unwilling to read English materials. They cannot read and comprehend the English literatures, nor do learn the new knowledge by autonomous learning. Although some college English teachers spend much time on the training of reading ability, the effect is often trivial. The students often have some misunderstandings on the training of reading ability. They believe that increasing vocabularies and remembering grammar rules can improve the efficiency of reading. But this opinion is naive. The survey shows that this method cannot improve the reading comprehension ability for the students. How to improve the English reading ability becomes the focus of the reform of college English teaching. The reading teaching of college English based on schema theory can well solve these problems and rapidly improves the reading ability of students.

\section{CONCEPTS OF SCHEMA THEORY}

The word 'schema' comes from Greek language and is a term of cognitive psychology. In 1781, this concept is first proposed by a German philosopher named Kant. In 1932, British psychologist Bartlett developed the concept of schema and applied it to the study of psychology. He held that schema is an active organization of the previous reaction or experience. It is the process that the information stored in the brains of learners reacts to the new information and the new information is absorbed to the knowledge base of learners.[1] Until 1980s, Rumelhart, an American famous expert on artificial intelligence, developed the theory and completed the system. He held that schema is the interacting knowledge structure or the building blocks of constructing the cognitive ability that store in the brains of learner in the hierarchy forms. The schema theory is also the theoretical model that is used to systematically and deeply study the effect of long-time memory in the process of understanding.[2] Although different disciplines have different understandings perspectives on the schema, we can conclude easily that schema is the unit that represents the previous knowledge and experience and the new information organized and stored in the brains of human beings. Schema is the knowledge structure that exists in the human brains. It consists of slots. The process of filling the slots is the process of schema concretion. When the slots of schema are filled, the schema is activated. Rumelhart held that understanding is the process that the brain selects the corresponding schema and the variables are under constraint. According to this view, a certain amount of information must be input to the reader in the process of reading comprehension. Then the reader begins to search for the schema that can explain the information. When the schema is found, it is activated and the article is understood effectively.

The schema theory emphasizes that the cognitive activity must depend on the previous knowledge structure in the brains. The theory held that reading is the process that the background knowledge of students interacts with the information of reading materials. In the process of reading, the background knowledge can help the student forecast the information and effectively comprehend the article. Meanwhile, as the student goes on decoding the article, he or she continues to correct and supplement the information predicated. Even though the readers have the same language level, the reader that has abundant schemas will perform better in understandings and memory.[3]

There are three kinds of schemas: the linguistic schema, the format schema and the content schema. Linguistic schema is about the language knowledge of vocabulary, grammar, syntax and usage. It is the ability that the reader masters and uses the language. The language knowledge is the prerequisite that the reader understands the discourse. When the students lack too much language knowledge, e.g. many unknown vocabularies or complex sentences in the article, they cannot decode the 
information via schema. Therefore, in the English reading teaching, teachers should pay attention to the situation of students' language knowledge and help them build rich language schemas. Format schema denotes the knowledge on structures and genre of different articles, such as drama, scientific articles, essays, and so on. These articles have their own unique structures. The survey shows that the narrative writing with the chronological structure and the descriptive writing with the unidirectional structure are easier to recall than the writing with other structures. This means that the knowledge of article structure can increase the comprehension on article content for the readers. Therefore, in the teaching of reading, teacher should instruct the students to acquaint the format schemas in order to understand the contents and structures of articles. If the discourse knowledge can effectively activate the relevant format schemas, the students can grasp the main idea and the logical relationship between the paragraphs and form a correct foresight further. This can improve the efficiency of reading. Content schema refers to the background knowledge on the reading materials for the readers. It also includes the cultural background knowledge. Content schema can directly affect the comprehension of readers on the subject, and therefore it plays an irreplaceable role in reading comprehension. Generally, the more the readers have the content schemas, the greater the readers have the probability to understand the article. In other words, if the readers are familiar with the background knowledge of reading materials, the readers can better understand the articles. In some extent, rich background knowledge can also compensate the readers for the lack of language knowledge. It can help the readers to forecast the information and eliminate the ambiguity, and thus can deepen the understanding of the article. [4]

Schema theory holds that reading can be divided into three modes. One is the bottom-up mode of Gough. It means that starting from the smallest unit, e.g. phoneme, morpheme, word, etc, the readers confirm the meaning of words by decoding. Then the readers modify the related knowledge in brains as well as the predicted information, and consequently draw the correct explanations. Therefore, in the traditional language teaching of reading comprehension, teacher always follows the order of presentation, that is, the teacher first introduces new words, and then explain the phrases and grammar to the students, and then asks the student to do exercises.[5] But the scholars have great dispute on this mode because this mode assumes that reading is the single process to obtain meaning from reading materials. Many studies found that existing knowledge of the readers have great influence on comprehension. Therefore, the traditional teaching has low efficiency and lots of problems. For instance, it emphasizes the accuracy of reading, but ignores the reading speed. It pays much attention to words and sentences while neglecting the overall significance. The second mode is the top-down mode proposed by Goodman. It means that the readers start from the high-level information and make assumptions on the article according to their existing knowledge, and then understand the article in specific aspects and confirm the speculation or hypothesis. Goodman believes that reading is the process of persistent speculation and confirmation, and the readers make verifications while speculating according to the obtained information. The theoretical mode of Goodman emphasizes the role of existing knowledge of readers in the process of reading. It believes that the reading material is nothing but to provide a few hints to the readers. Effective reading is not that readers understand the materials verbatim, but that the readers can speculate the contents of article. This is a great breakthrough in fast reading teaching. But this mode has gone into an extreme and has its own problems. It too emphasizes the top-down process while ignoring the bottom-up process. In the teaching practice, this mode often neglects the accumulation of knowledge in the reading comprehension. The third mode is the interactive mode proposed by Rumelhart. He absorbed the latest achievements in the field of cognition and held that reading is the process of multi languages interact complicatedly. Based on the studies of Gough and Goodman, Rumelhart proposed that reading is the process that the visual information interacts with the existing knowledge of readers.

\section{StRATEGY OF READING TEACHING BASED ON SCHEMA THEORY}

Now we know that reading is a complex cognitive process. In college English reading teaching, application of schema theory not only promotes the understanding of content, but also improves the reading efficiency of students. Teacher not only helps the students to decode the content, but also activates the schemas stored in the brains of students and guides the students to comprehend the discourse and actively construct new schemas.

(i) Make full use of speculating role of schema and activate the existing schemas of students. In reality, we do not need to experience everything and we can know the existence of lots of things. For instance, when mentioning the lamp, we naturally know that it has the switch. This is because everything in the world has its stereotype and this rule is universal. Therefore, when a certain schema is determined in the reading process, the readers assume the slots of schema conform to their experience. Therefore, in order to erase the obstacles in reading comprehension, the teacher purposely organizes the students to prepare for reading and activates their schemas stored in brains and instructs the students to speculate the content of article via the bottom-up and top-down modes. The methods of teaching can be flexible and diverse, such as slide show, watching video, keywords prompt etc. The purpose is to activate the existing schemas of students and help them to construct new schemas and ultimately improve the reading efficiency.

(ii) Help the students to build rich content schema. Content schema embodies the proficiency of students on the topics. When the students lack the content schemas, they cannot be unable to effectively understand the article even if they have much knowledge of language. Therefore, it is important to help the students build rich content schemas. In teaching of reading, the teacher should expand the knowledge of students purposely and introduces more background knowledge of western culture, such as religious beliefs, customs and habits, historical background and other related knowledge. Before the class training, the teacher should introduce the relevant knowledge of cultural background to the students first and encourage the students to compare the culture background of their own with the cultures of other nations. This can facilitate the formation and memory of schemas for students. Then the teacher asks the 
students to read the according articles and activates the schemas of students. This can strengthen the comprehension on the articles. At the same time, students can achieve more schema knowledge by reading. The previous schemas are modified and strengthened by new schemas and then the ability of reading on this aspect is improved.

(iii) Acquaint the structure difference between English and Chinese discourses and cultivate the awareness of cross-culture. Although English and Chinese have a lot in common, there are lots of differences in grammar representation and writing habits. Only after the students achieve the related schemas do they further understand the expression habits and structures of English articles. Therefore, the teacher should explain the difficult sentences and grammar usages to the students first and analyzes the modes of speaking and thinking and explain the writing habits of westerners. For example, the expression approach is much different between the articles of issue and argument. Generally, different opinions are summarized in the beginning of issue article and the author then analyzes them point by point and in the end of article the author presents his own opinion on the issue. In argument, the author first summarizes the controversial views and then refutes the views point by point. The opinion of author is often shown in the refutations. For the scientific article, the author first gives his opinion and then demonstrates the opinion by series of proofs. The teacher should distinguish the structure difference between the articles and establishes the corresponding schemas for the students. Then the schemas are activated in the training of reading and this can enhance the comprehension ability of students.

(iv) Apply the schema theory to language teaching and cultivate the reading skills. It is important for the teacher to help the students build rich schemas in reading training. Nevertheless, it is also important to activate and strengthen the schemas by training of reading. If the schemas are not activated, the students can not well understand the reading materials. Therefore, the teacher should make a detailed plan for the students to improve the reading ability. The teacher should classify the articles according to the themes and formats and then guides the students to participate in special training. The teacher should first instruct the students in knowledge of reading and helps them build the schemas, and then strengthens the schemas via reading comprehension. In the reading teaching, the teacher should encourage the students to summarize the writing format and grammar knowledge, and guides the students to speculate the purpose of author via the schema knowledge. This can eventually make the students to read actively. In the aspect of cultural background, the teacher should introduce the familiar background knowledge to students and encourages the students to compare it with other cultures and eventually expands the knowledge scope of students. Moreover, the teacher should demonstrate to the students how to speculate the purpose of author via the keywords in article. By these training, the students can read articles effectively and fast.

\section{CONCLUSION}

Schema theory is the theory of second language acquisition based on constructivism. It can guide the learning of students effectively. It is the focus of reform of English teaching how to apply the schema theory to the teaching of reading comprehension. It can greatly improve the reading ability of students via building and application of schema.

\section{ACKNOWLEDGEMENT}

This work is part of the project of Research on Task-based English Teaching Mode Based on Constructivism Theory. The work has been supported from the fund of Liaoning Provincial Association of Social Sciences (No. 2015lslktziwx-03).

\section{REFERENCES}

[1] Eysenck M.W., Keane M.T., Cognitive Psychology: a Student's Handbook, Lawrence Erlbaum. 1990.

[2] Carrell P.L., Evidence of a formal schema in second language comprehension, Language Learning, 33, 83-203, 1984.

[3] Carrell P.L., Eisterhold J.C., Schema theory and ESL reading pedagogy, TESOL Quarterly, 17, 553-573, 1983.

[4] Adams M.J., Collins A., A Schema-theoretic view of reading. New Jersey: Ablex Publishing Corporation, 1979.

[5] Clapham C., The Development of IELTS: A Study of the Effects of Background Knowledge on Reading Comprehension. Cambridge: Cambridge University Press. 1996. 